\title{
A Crank-Nicolson Scheme for the Dirichlet-to-Neumann Semigroup
}

\author{
Rola Ali Ahmad, ${ }^{1}$ Toufic El Arwadi, ${ }^{1}$ Houssam Chrayteh, ${ }^{1}$ and Jean-Marc Sac-Epée ${ }^{2}$ \\ ${ }^{1}$ Department of Mathematics, Faculty of Science, Beirut Arab University, P.O. Box 11-5020, Beirut, Lebanon \\ ${ }^{2}$ Institut Élie Cartan de Lorraine, UMR 7502, Université de Lorraine, 57045 Metz, France
}

Correspondence should be addressed to Jean-Marc Sac-Epée; jean-marc.sac-epee@univ-lorraine.fr

Received 4 May 2015; Accepted 28 June 2015

Academic Editor: M. Montaz Ali

Copyright (c) 2015 Rola Ali Ahmad et al. This is an open access article distributed under the Creative Commons Attribution License, which permits unrestricted use, distribution, and reproduction in any medium, provided the original work is properly cited.

\begin{abstract}
The aim of this work is to study a semidiscrete Crank-Nicolson type scheme in order to approximate numerically the Dirichletto-Neumann semigroup. We construct an approximating family of operators for the Dirichlet-to-Neumann semigroup, which satisfies the assumptions of Chernoff's product formula, and consequently the Crank-Nicolson scheme converges to the exact solution. Finally, we write a $P_{1}$ finite element scheme for the problem, and we illustrate this convergence by means of a FreeFem++ implementation.
\end{abstract}

\section{Introduction}

Let $\Omega$ be a bounded smooth domain $\Omega \subset \mathbb{R}^{n}$ and let $\gamma(x)=\left[\gamma_{i, j}(x)\right]_{i, j=1}^{n}$ be a real-valued matrix function, which is known as the electrical conductivity matrix. The matrix $\gamma(x)$ is symmetric and smooth; that is, $\gamma_{i j}=\gamma_{j i} \in C^{\infty}(\bar{\Omega})$. Its positive eigenvalues are uniformly bounded; that is, there exists $0<c_{1}<c_{2}$ such that, for all $(x, \xi) \in \Omega \times \mathbb{R}^{n}$, one has $c_{1}\|\xi\|^{2} \leq \xi^{T} \gamma(x) \xi \leq c_{2}\|\xi\|^{2}$, where $\|\cdot\|$ is the Euclidean norm of $\mathbb{R}^{n}$.

In this paper, we denote by $X=L^{2}(\Omega)$, with boundary space $\partial X=L^{2}(\partial \Omega)$. It is well known that, for any $f \in$ $H^{1 / 2}(\partial \Omega)$, the following Dirichlet problem,

$$
\begin{aligned}
\operatorname{div}(\gamma \nabla u) & =0, & & \text { in } \Omega, \\
u & =f, & & \text { on } \partial \Omega,
\end{aligned}
$$

has a unique solution $u=L_{\gamma} f$ in $H^{1}(\Omega)$, which is called the $\gamma$-harmonic lifting of $f$; see [1] for more details.

Define the action of the Dirichlet-to-Neumann operator on $f$ as the outward normal derivative of $u$ on the boundary; that is,

$$
\Lambda_{\gamma}(f):=\left.(\nu \cdot \gamma \nabla u)\right|_{\partial \Omega},
$$

where $\nu$ is the outer normal vector to $\partial \Omega$ at $x \in \partial \Omega$.
This operator is defined on its domain:

$$
D\left(\Lambda_{\gamma}\right):=\left\{f \in H^{1 / 2}(\partial \Omega) ; \Lambda_{\gamma} f \in L^{2}(\partial \Omega)\right\} .
$$

The Dirichlet-to-Neumann semigroup is the trace of the following boundary value problem:

$$
(\mathrm{BVP}) \begin{cases}\operatorname{div}(\gamma \nabla u(t, \cdot))=0, & \text { in } \Omega, \\ \partial_{t} u+\nu \cdot \gamma \nabla u=0, & \text { on } \partial \Omega, \\ u(0, \cdot)=f, & \text { on } \partial \Omega .\end{cases}
$$

In [1], Lax has introduced a semigroup in $\partial X$, where $\Omega$ is the unit ball $B(0,1)$ and $\gamma$ is the identity matrix. Let $v$ be the solution of the problem:

$$
\begin{array}{ll}
\Delta v=0, & \text { in } \Omega, \\
L v=f, & \text { on } \partial \Omega,
\end{array}
$$

where $L$ is the trace operator on $\partial \Omega$. The Lax semigroup is defined by

$$
S(t)=L T(t) L_{0},
$$

where $L_{0}$ is the lifting operator and $T(t) v(x):=v\left(e^{-t} x\right)$. 
Therefore, if $f \in \partial X$, then, for any $w \in \partial \Omega$,

$$
(S(t) f)(w)=v\left(e^{-t} w\right) .
$$

It has been shown that the Lax semigroup is actually the Dirichlet-to-Neumann semigroup.

In [2], it is shown that Lax's representation cannot be generalized if $\Omega$ is not the unit ball. This was a motivation for many authors to use Chernoff's formula in order to approximate the solution.

In [3], Emamirad and Shariftabar proved that the Euler Explicit scheme

$$
\begin{aligned}
\operatorname{div}\left(\gamma \nabla u^{m+1}\right) & =0, & & \text { in } \Omega, \\
\frac{1}{\Delta t}\left(u^{m+1}-u^{m}\right)+\frac{\partial u^{m}}{\partial v_{\gamma}} & =0, & & \text { on } \partial \Omega, \\
u^{0} & =f, & & \text { on } \partial \Omega
\end{aligned}
$$

converges to the solution of the (BVP).

Later, Cherif et al. proposed, in [4], the following Implicit Euler Scheme:

$$
\begin{aligned}
\operatorname{div}\left(\gamma \nabla u^{m+1}\right) & =0, & & \text { in } \Omega, \\
\frac{1}{\Delta t}\left(u^{m+1}-u^{m}\right)+\frac{\partial u^{m+1}}{\partial \nu_{\gamma}} & =0, & & \text { on } \partial \Omega, \\
u^{0} & =f, & & \text { on } \partial \Omega,
\end{aligned}
$$

which converges to the solution of the (BVP).

After studying the implicit and explicit schemes, by the same strategy, we are interested in studying the convergence of the Crank-Nicolson type scheme. This paper is divided into 4 sections, as follows.

First, in Section 2, we introduce the Crank-Nicolson type scheme for evolution equations.

In Section 3, we prove the convergence of a CrankNicholson type scheme for the (BVP), by constructing an approximating family, and use Chernoff's product formula in order to prove the convergence in $L^{2}(\partial \Omega)$.

Finally, in Section 4, we present a numerical implementation of the Crank-Nicolson type scheme in the computational framework of the finite element method.

\section{The Crank-Nicolson Scheme}

In numerical analysis, the Crank-Nicolson method is a finite difference method used for numerically solving the heat equation and similar partial differential equations. It is a second-order method in time and it is numerically stable. The method was developed by John Crank and Phyllis Nicolson in the mid-20th century.

Let $A$ be a $m$-accretive operator on the Hilbert space $H$ defined as $A: D(A) \rightarrow H$.

Consider the evolution problem:

$$
\begin{gathered}
\frac{\partial u}{\partial t}+A u=0, \quad \text { for } 0<t<T, \\
u(0, x)=u_{0}(x) .
\end{gathered}
$$

We approximate the time derivative by using the finite difference method as follows:

$$
\begin{aligned}
u(n \Delta t) & \approx u^{n} \\
\frac{\partial u}{\partial t}(n \Delta t) & =\frac{1}{\Delta t}(u(n+1) \Delta t-u(n \Delta t)) \\
& \approx \frac{1}{\Delta t}\left(u^{n+1}-u^{n}\right), \quad \text { for } n=0, \ldots,\left[\frac{T}{\Delta t}\right] .
\end{aligned}
$$

Crank-Nicolson introduced the scheme:

$$
\begin{array}{r}
\frac{u^{n+1}-u^{n}}{\Delta t}+\frac{1}{2} A u^{n}+\frac{1}{2} A u^{n+1}= \\
\quad \text { for } n=0, \ldots,\left[\frac{T}{\Delta t}\right], \\
u_{0} \quad \text { given. }
\end{array}
$$

The Chernoff product formula holds not only in the case of semigroup of contractions but also if the semigroup is bounded, so that the Crank-Nicholson scheme for the (BVP) can be written as

$$
\text { (CNS) } \begin{cases}\operatorname{div}\left(\gamma \nabla u^{m+1}\right)+\operatorname{div}\left(\gamma \nabla u^{m}\right)=0, & \text { in } \Omega, \\ \frac{1}{\Delta t}\left(u^{m+1}-u^{m}\right)+\frac{1}{2}\left(\frac{\partial u^{m}}{\partial \nu_{\gamma}}+\frac{\partial u^{m+1}}{\partial \nu_{\gamma}}\right)=0, & \text { on } \partial \Omega, \\ u^{0}=f, & \text { on } \partial \Omega .\end{cases}
$$

\section{Convergence of the Crank-Nicholson Type Scheme}

In order to show the convergence of the (CNS), let us recall the following Chernoff theorem.

Theorem 1 (Chernoff's product formula). Let $X$ be a Banach space, real or complex, and let $\{V(t) ; t \geq 0\}$ be a family of contractions on $X$ with $V(0)=I$.

Assume that there exists $A: D(A) \subseteq X \rightarrow X$ which generates a $C_{0}$-semigroup of contractions $\{S(t) ; t \geq 0\}$ and that for all $x \in D(A)$ there exists

$$
\lim _{h \rightarrow 0} \frac{1}{h}(V(h)-I) x=A x .
$$

Then, for each $x$ in $X$, one has $\lim _{n \rightarrow \infty} V^{n}(t / n) x=S(t) x$.

Remark 2. Note that Chernoff's product formula holds not only in the case of semigroup of contractions but also if the semigroup is bounded (see [5]).

This theorem was used to prove the convergence of both implicit and explicit schemes.

Now, in order to prove the convergence of the CrankNicolson scheme, we will show that the operator $Z(t)$, defined below, satisfies the 3 conditions of Chernoff's theorem. 
For any $x \in \partial \Omega$, we have

$$
\begin{aligned}
\frac{\partial u^{m+1}}{\partial \nu_{\gamma}} & =\frac{1}{\Delta x}\left[u^{m+1}(x)-u^{m+1}(x-\Delta x \gamma(x) \nu)\right], \\
\frac{\partial u^{m}}{\partial \nu_{\gamma}} & =\frac{1}{\Delta x}\left[u^{m}(x)-u^{m}(x-\Delta x \gamma(x) \nu)\right] .
\end{aligned}
$$

Using in (CNS) the approximation given in (15), we get

$$
\begin{aligned}
{[1+} & \left.\frac{1}{2} \frac{\Delta t}{\Delta x}\right] u^{m+1}(x)-\left[1-\frac{1}{2} \frac{\Delta t}{\Delta x}\right] u^{m}(x) \\
& -\frac{1}{2} \frac{\Delta t}{\Delta x} u^{m+1}(x-\Delta x \gamma(x) \nu) \\
& -\frac{1}{2} \frac{\Delta t}{\Delta x} u^{m+1}(x-\Delta x \gamma(\nu))=0 .
\end{aligned}
$$

Taking $\alpha=\Delta t / \Delta x$, we get

$$
\begin{aligned}
& {\left[1+\frac{\alpha}{2}\right] u^{m+1}(x)-\frac{\alpha}{2} u^{m+1}(x-\Delta x \gamma(x) \nu)} \\
& =\left[1-\frac{\alpha}{2}\right] u^{m}(x)+\frac{\alpha}{2} u^{m+1}(x-\Delta x \gamma(x) \nu)
\end{aligned}
$$

which can be written as

$$
W(\Delta t) u^{m+1}=V(\Delta t) u^{m}
$$

where

$$
\begin{aligned}
& W(\Delta t) f=\left[1+\frac{\alpha}{2}\right] u-\frac{\alpha}{2} u\left(x-\alpha^{-1} t \gamma(x) \nu\right), \\
& V(\Delta t) f=\left[1-\frac{\alpha}{2}\right] u+\frac{\alpha}{2} u\left(x-\alpha^{-1} t \gamma(x) \nu\right)
\end{aligned}
$$

Now, we define the family $Q(t)$ as the inverse of $W(t)$ in the following sense:

$$
W(\Delta t) Q(\Delta t) f(x)=f(x)
$$

and we show that $Z(t)=Q(\Delta t) V(\Delta t)$ satisfies all the assumptions of Chernoff's product formula.

(i) $Z(0) f=f$. For any $x \in \partial \Omega$, we have

$$
\begin{aligned}
W(0) f(x) & =\left(1+\frac{\alpha}{2}\right) u(x)-\frac{\alpha}{2} u(x)=u(x) \\
& =f(x)
\end{aligned}
$$

so, $f(x)=W(0)[Q(0) f(x)]=Q(0) f(x)$.

Moreover,

$$
\begin{aligned}
V(0) f(x) & =\left(1-\frac{\alpha}{2}\right) u(x)+\frac{\alpha}{2} u(x)=u(x) \\
& =f(x)
\end{aligned}
$$

so,

$$
V(0) f(x)=f(x) .
$$

Using (6) and (7), we get

$$
W(0) f(x)=V(0) f(x) .
$$

Therefore, $f(x)=Q(0) V(0) f(x)=Z(0) f(x)$. (ii) $Z^{\prime}(0) f=-\Lambda_{\gamma} f$. The derivative of $W(t)$ with respect to $t$ is

$$
W^{\prime}(t) f=-\frac{\alpha}{2}\left(-\alpha^{-1} \gamma \nu\right) \cdot \nabla u\left(v-\alpha^{-1} t \gamma \nu\right)
$$

At point $t=0$, we have $W^{\prime}(0) f=1 / 2 \Lambda_{\gamma} f$.

Similarly, the derivative of $V(t)$ with respect to $t$ is

$$
V^{\prime}(t) f=\frac{\alpha}{2}\left(-\alpha^{-1} \gamma \nu\right) \cdot \nabla u\left(\nu-\alpha^{-1} t \gamma \nu\right)
$$

At point $t=0$, we have $V^{\prime}(0) f=-(1 / 2) \Lambda_{\gamma} f$.

But $W(t) Q(t) f=f$, so $W^{\prime}(t) Q(0) f+W(0) Q^{\prime}(0) f=0$, and then $Q^{\prime}(0) f=-(1 / 2) \Lambda_{\gamma} f$.

Now, the derivative of $Z(t)$ with respect to $t$ is

$$
Z^{\prime}(t) f=Q^{\prime}(t) V(t) f+Q(t) V^{\prime}(t) f .
$$

At $t=0, Z^{\prime}(0) f=Q^{\prime}(0) V(0) f+Q(0) V^{\prime}(0) f=-\Lambda_{\gamma} f$.

Therefore,

$$
Z^{\prime}(0) f=\Lambda_{\gamma} f
$$

(iii) $Z(t)$ Is a Contraction. In fact,

$$
\begin{aligned}
& \|W(t) f\|_{L^{2}(\partial \Omega)}^{2} \\
& \quad=\int_{\partial \Omega}\left|u(x)+\frac{\alpha}{2}\left(u(x)-u\left(x-\alpha^{-1} t \gamma \nu\right)\right)\right|^{2} d \sigma
\end{aligned}
$$

but $\left(u(x)-u\left(x-\alpha^{-1} t \gamma \nu\right)\right) / \alpha^{-1}$ is an upper bound of the normal derivative $\partial u / \partial v_{\gamma}$, so that this term is positive according to Hopf's lemma, and, consequently,

$$
\begin{gathered}
\int_{\partial \Omega}\left|u(x)+\frac{\alpha}{2}\left(u(x)-u\left(x-\alpha^{-1} t \gamma \nu\right)\right)\right|^{2} d \sigma \\
\geq \int_{\partial \Omega}|u(x)|^{2} d \sigma=\|f\|_{L^{2}(\partial \Omega)}^{2}
\end{gathered}
$$

which implies that

$$
\|W(t) f\|_{L^{2}(\partial \Omega)} \geq\|f\|_{L^{2}(\partial \Omega)} .
$$

Now

$$
\|f\|_{L^{2}(\partial \Omega)}=\|W(t) Q(t) f\|_{L^{2}(\partial \Omega)} \geq\|Q(t) f\|_{L^{2}(\partial \Omega)} .
$$

Thus, $Q(t)$ is of contraction.

Similarly $V(t)$ is also of contraction.

Finally,

$$
\begin{aligned}
\|Z(t) f\|_{L^{2}(\partial \Omega)} & =\|Q(t) V(t) f\|_{L^{2}(\partial \Omega)} \\
& \leq\|Q(t) f\|_{L^{2}(\partial \Omega)} \leq\|f\|_{L^{2}(\partial \Omega)} .
\end{aligned}
$$

Therefore, $Z(t)$ is of contraction.

So, we have proved that $Z(t)$ satisfies all the assumptions of Chernoff's product formula, and, consequently, the CrankNicholson scheme converges to its exact solution.

Theorem 3. The operator $Z(t)$ satisfies the Chernoff conditions; hence, the Crank-Nicolson method is convergent. 


\section{Numerical Results}

In the last section of this paper, we present the resolution of (CNS) in the particular case where $\gamma$ is the unit matrix and $\Omega$ is the open unit disk. The point in dealing with this case is that the exact solution is known to be $v\left(e^{-t} x, e^{-t} y\right)$, where $v$ is the solution of the Laplace equation $\Delta v=0$, with $v=u_{0}$ on the boundary ( $u_{0}$ is any regular function). Thus, we can compare this exact solution with the approximate solution obtained via our Crank-Nicolson scheme. For this test, we choose $u_{0}(x, y)=0.5\left(x^{2}-y^{2}\right)+y+0.5$. The variational formulation of the (CNS) can be derived by multiplying both sides of the problem by a test function $v$ :

$$
\int_{\Omega} \gamma \Delta u^{m+1} v d x+\int_{\Omega} \gamma \Delta u^{m} v d x=0
$$

Using Green's formula, we get

$$
\begin{gathered}
-\int_{\Omega} \gamma \nabla u^{m+1} \nabla v d x-\int_{\partial \Omega} \frac{2}{\Delta t}\left[u^{m+1}-u^{m}\right] v d \sigma \\
-\int_{\Omega} \gamma \nabla u^{m} \nabla v d x=0 .
\end{gathered}
$$

Multiplying by $\Delta t$, we get

$$
\begin{gathered}
\int_{\Omega} \Delta t \gamma \nabla u^{m+1} \nabla v d x+2 \int_{\partial \Omega} u^{m+1} v d \sigma \\
=-\int_{\Omega} \Delta t \gamma \nabla u^{m} \nabla v d x+2 \int_{\partial \Omega} u^{m} v d \sigma
\end{gathered}
$$

which is of the form

$$
a\left(u^{m+1}, v\right)=l(v)
$$

where the bilinear form

$$
\begin{gathered}
a\left(u^{m+1}, v\right)=\int_{\Omega} \Delta t \gamma \nabla u^{m+1} \nabla v d x+2 \int_{\partial \Omega} u^{m+1} v d \sigma, \\
l(v)=-\int_{\Omega} \Delta t \gamma \nabla u^{m} \nabla v d x+2 \int_{\partial \Omega} u^{m} v d \sigma
\end{gathered}
$$

is the linear form.

Using this variational formulation, the problem can be approximate via $P_{1}$-finite elements implemented in Freefem ++ .

The actual algorithm is as follows.

Choose an initial $u_{0}$ and, for a given $\Delta t$, where $t<N \Delta t$, we have the following:

for $(t=0 ; t<N \Delta t)$ do

(i) $u^{n+1}=Z\left(u^{n}\right)$

(ii) compute $u^{n+1}$ via the variational problem

(iii) $n \leftarrow n+1$

end do.

As an illustration, let us represent the exact (Figure 1) and the approximate solution (Figure 2), the $L^{2}$-error between the exact and the approximate solutions (Figure 3), and the decrement of this $L^{2}$-error according to the fineness of the meshing (Figure 4).

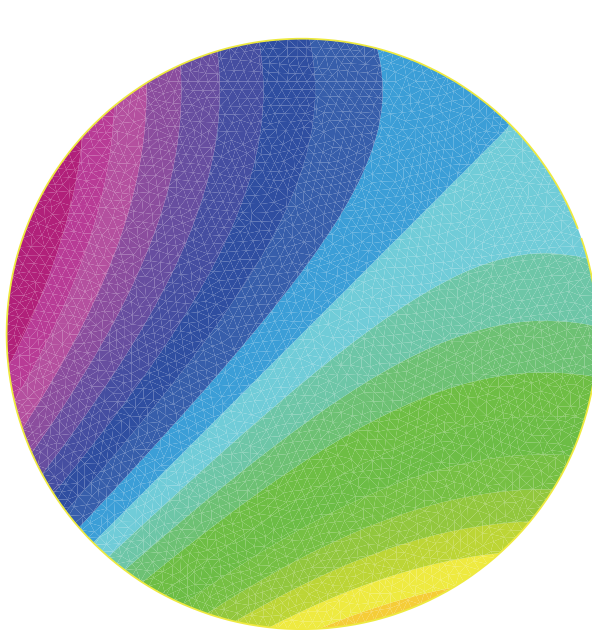

Iso value

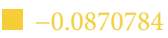

$-0.00320263$

0.0527145

0.108632

0.164549

0.220466

0.276383

0.3323

0.388218

0.444135

0.500052

0.555969

— 0.611886

— 0.667803

- 0.723721

ㅁ.779638

- 0.835555

ㅁ 0.891472

$\square .947389$

- 1.08718

Figure 1: The exact solution.

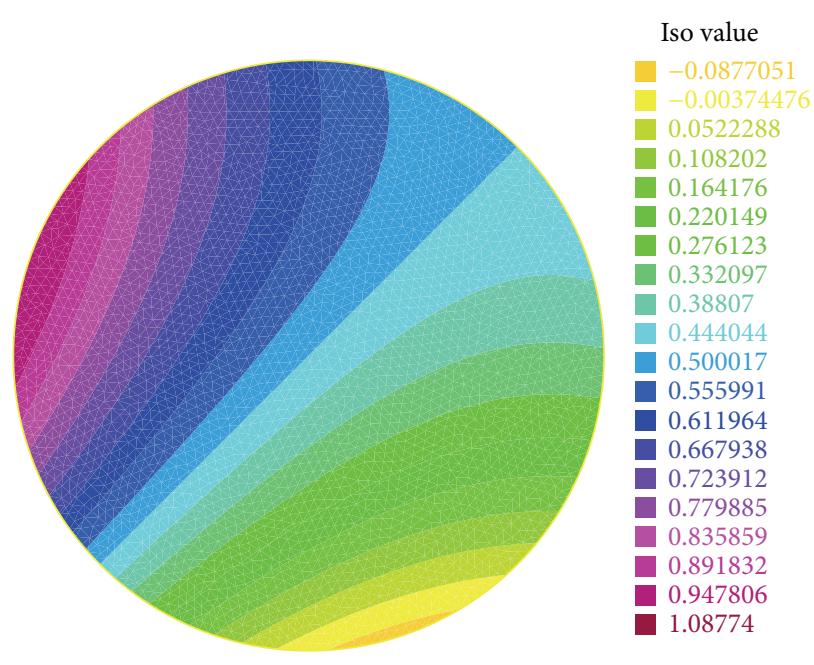

Figure 2: The numerical solution.

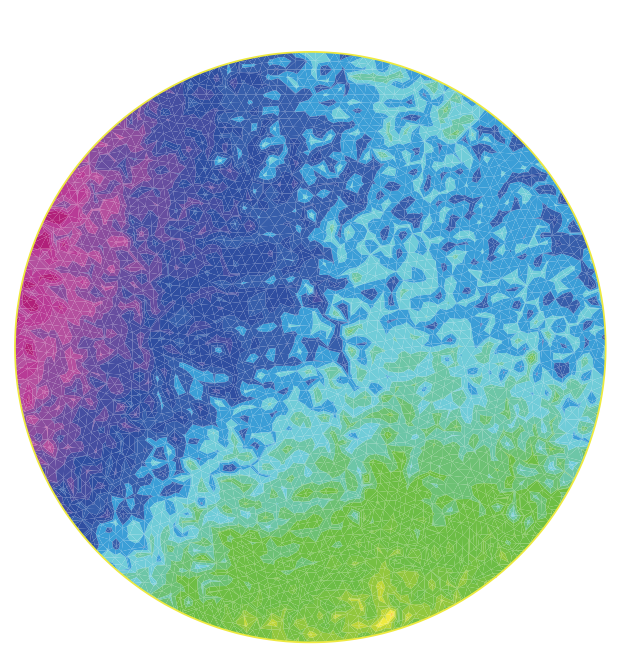

Iso value

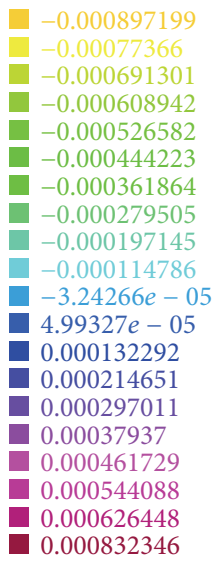

FIGURE 3: The difference between the exact and the numerical solutions. 


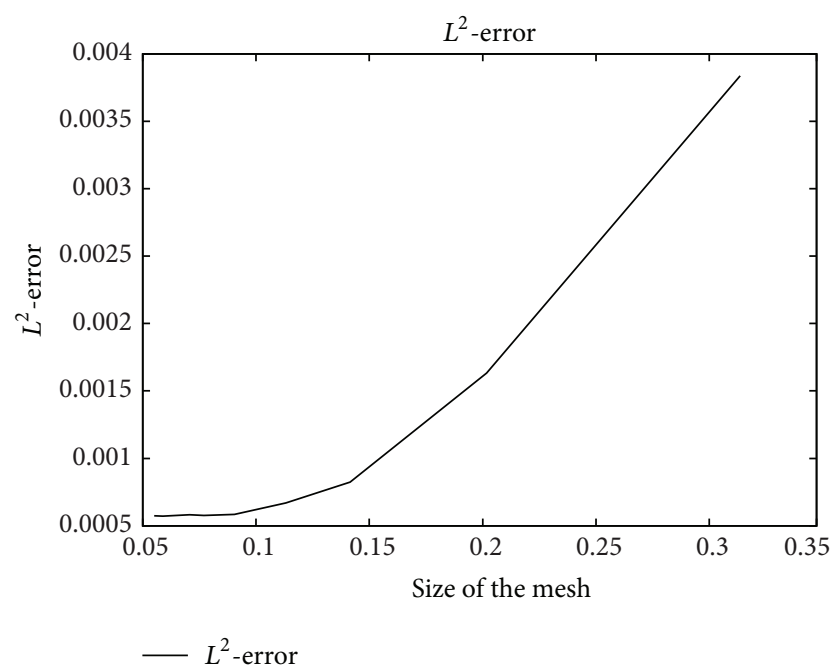

FIGURE 4: The $L^{2}$-error according to the fineness of the mesh.

\section{Conclusion}

As a conclusion, we have proved the convergence of the (CNS) by showing that the constructed family of operators satisfies the assumptions of Chernoff's product formula and consequently approximated numerically the Dirichlet-toNeumann semigroup.

\section{Conflict of Interests}

The authors declare that there is no conflict of interests regarding the publication of this paper.

\section{References}

[1] P. D. Lax, Functional Analysis, Wiley-Interscience, New York, NY, USA, 2012.

[2] H. Emamirad and I. Laadnani, "An approximating family for the Dirichlet-to-Neumann semigroup," Advances in Differential Equations, vol. 11, no. 3, pp. 241-257, 2006.

[3] H. Emamirad and M. Sharifitabar, "On explicit representation and approximations of Dirichlet-to-Neumann semigroup," Semigroup Forum, vol. 86, no. 1, pp. 192-201, 2013.

[4] M. A. Cherif, T. El Arwadi, H. Emamirad, and J.-M. Sac-épée, "Dirichlet-to-Neumann semigroup acts as a magnifying glass," Semigroup Forum, vol. 88, no. 3, pp. 753-767, 2014.

[5] K. Engel and R. Nagel, One-Parameter Semigroups for Linear Evolution Equations, vol. 194 of Graduate Texts in Mathematics, Springer, New York, NY, USA, 2000. 


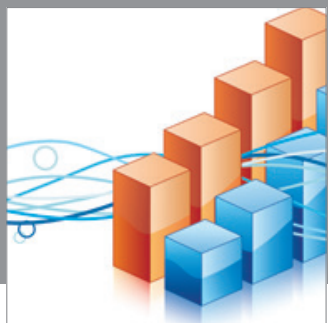

Advances in

Operations Research

mansans

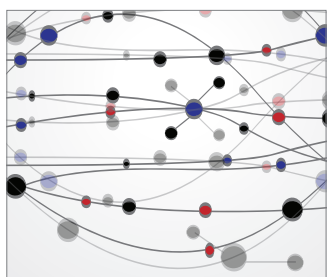

The Scientific World Journal
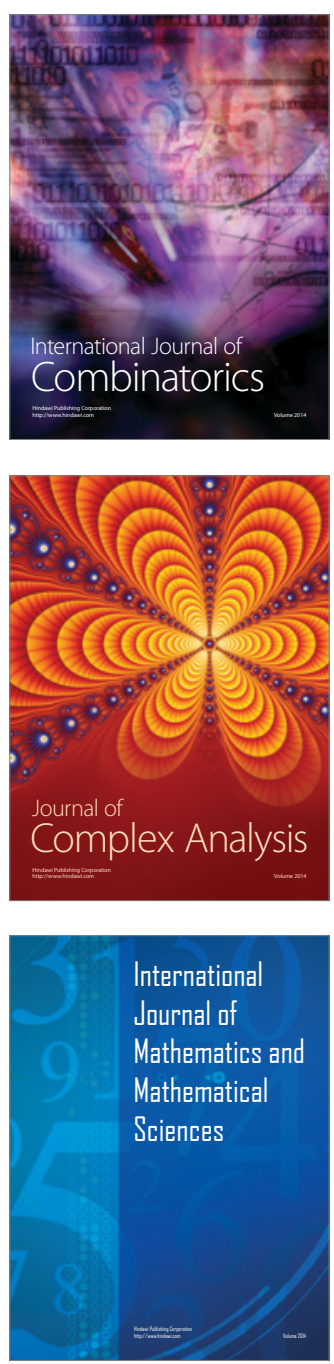
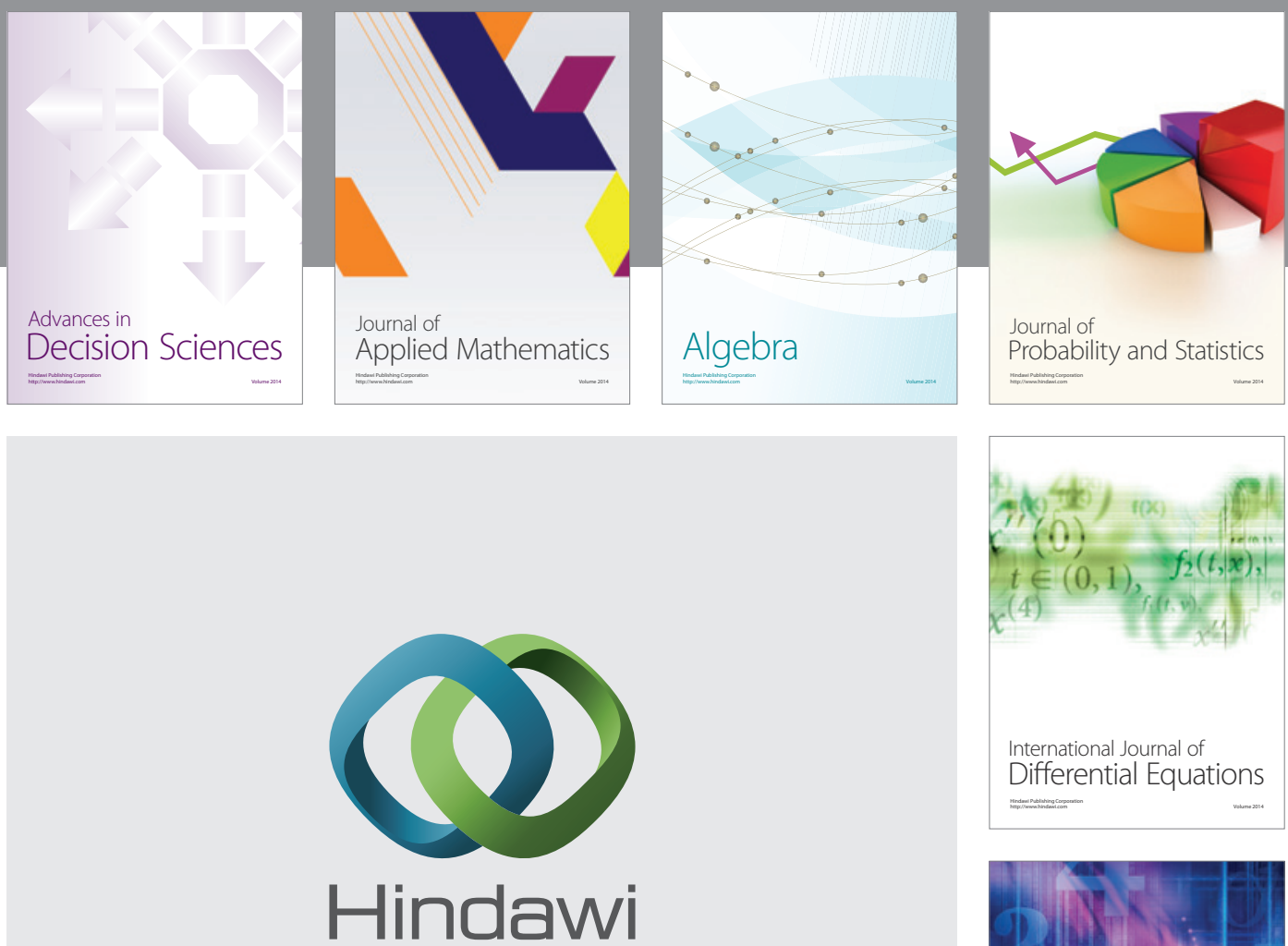

Submit your manuscripts at http://www.hindawi.com
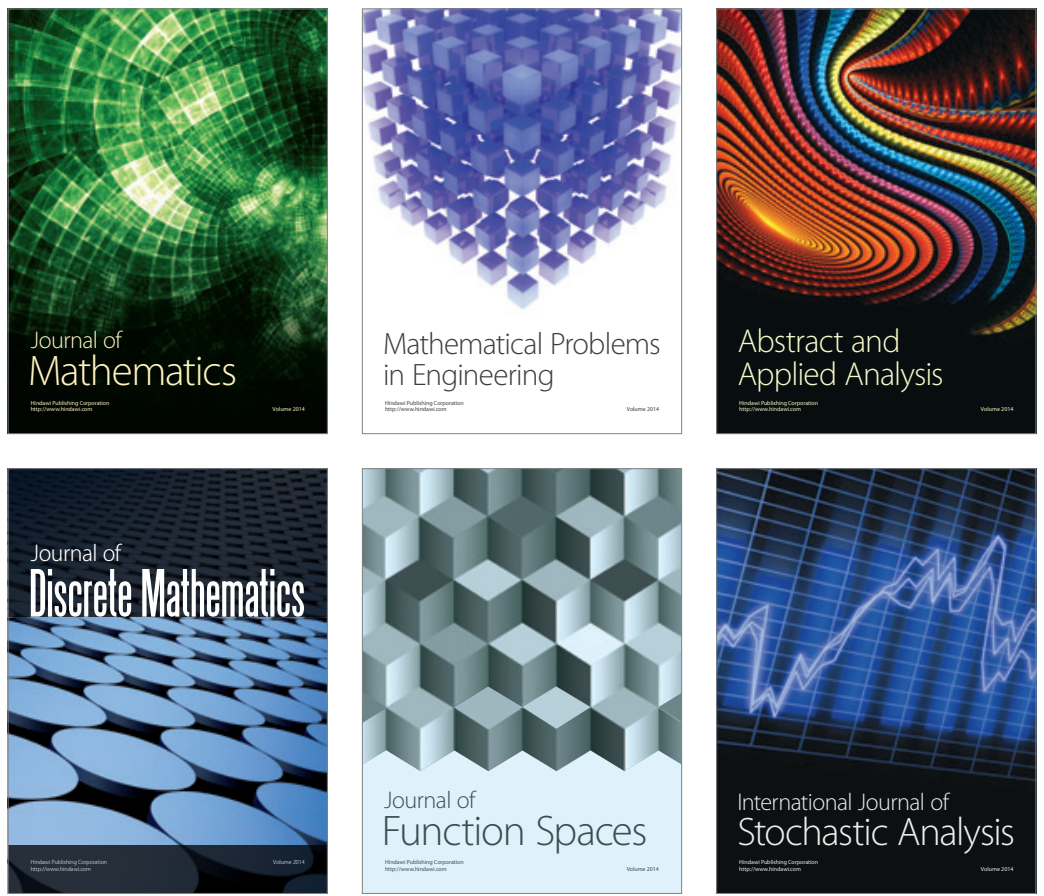

Journal of

Function Spaces

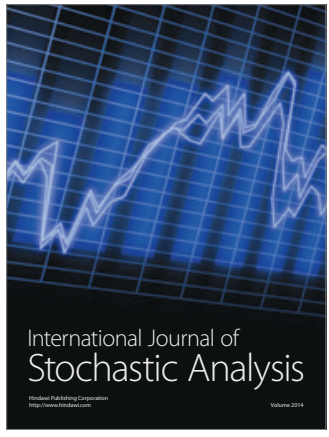

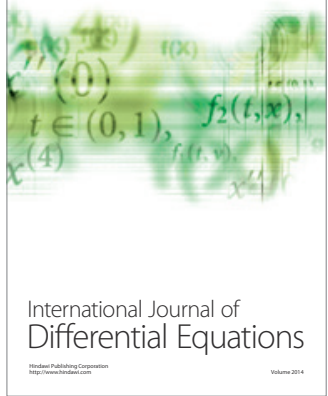
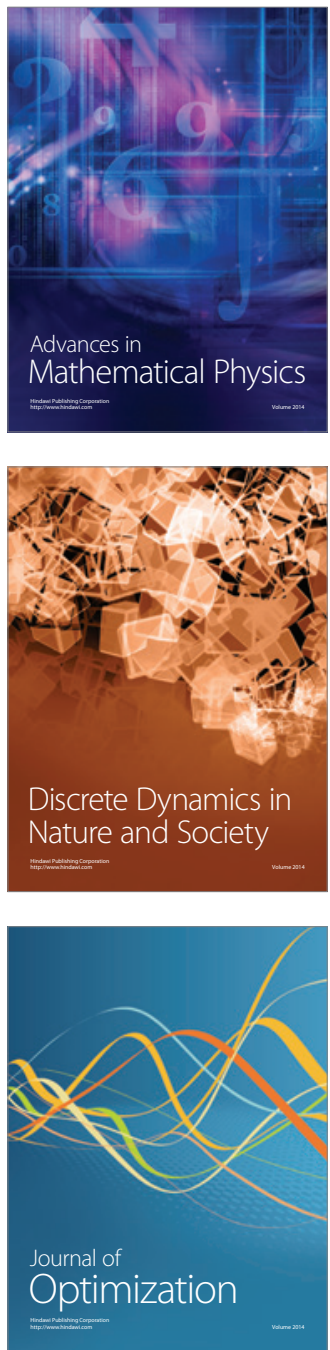